\title{
Loneliness among adolescents and young adults with cancer during the COVID-19 pandemic: a cross-sectional survey
}

\author{
Kaitlyn Howden ${ }^{1}$ - Adam P. Yan ${ }^{2}$. Camille Glidden ${ }^{3} \cdot$ Razvan G. Romanescu ${ }^{4}$ lan Scott ${ }^{5} \cdot$ Julie M. Deleemans $^{6}$. \\ Karine Chalifour $^{7}$. Geoff Eaton ${ }^{7}$. Abha A. Gupta ${ }^{1,8}$. James M. Bolton ${ }^{3,9}$. Sheila N. Garland ${ }^{10}$. Alyson L. Mahar ${ }^{11,12}$. \\ Sapna Oberoi ${ }^{1,12,13}$ (1)
}

Received: 19 July 2021 / Accepted: 12 October 2021 / Published online: 27 October 2021

(c) The Author(s), under exclusive licence to Springer-Verlag GmbH Germany, part of Springer Nature 2021

\begin{abstract}
Background Adolescents and young adults (AYAs) diagnosed with cancer are at an increased risk of experiencing social isolation and loneliness secondary to their cancer and its treatment. The physical distancing measures implemented during the COVID-19 pandemic may have further increased loneliness among this group. This study examined the prevalence of loneliness and factors associated with loneliness among AYAs with cancer during this pandemic.

Methods We conducted a self-administered, online, cross-sectional survey of Canadian AYAs diagnosed with cancer between 15 and 39 between January and February 2021. Loneliness was measured using the 3-item UCLA Loneliness Scale. Factors associated with higher levels of loneliness were identified using multiple logistic regression.

Results The analysis included 805 AYAs. The prevalence of loneliness was 52.2\% [ $N=419,95 \%$ CI (confidence interval) 48.7 to $55.6 \%$ ]. Individuals who were 18-25 years old [AOR (adjusted odds ratio)1.60, CI 1.03-2.47, $p=0.035$ ], currently undergoing cancer therapy (AOR 1.46, 95\% CI 1.03-2.07, $p=0.035$ ), who self-disclosed the presence of a pre-pandemic mental health condition (AOR 2.09, 95\% CI $=1.22-3.58, p=0.007$ ), or were not in a relationship (AOR 2.22, 95\% CI $1.57-3.14, p<0.001$ ) were more likely to report loneliness than others. Participants that lived in rural or remote locations were less likely to experience loneliness (AOR 0.59, 95\% CI 0.40-0.87, $p=0.008$ ).

Conclusion One in two AYAs with cancer are feeling lonely during the COVID-19 pandemic. Future studies for developing interventions to target loneliness, particularly for those at greater risk, are necessary to improve the health and quality of life of AYAs with cancer.
\end{abstract}

Keywords Social isolation · COVID-19 · Adolescents and young adults · Cancer · Oncology

\section{Introduction}

COVID-19 is a novel coronavirus responsible for a worldwide pandemic that has resulted in millions of deaths and has caused significant changes to the social landscape by implementing physical distancing practices [1-4]. In March 2020, the COVID-19 pandemic was declared a state of emergency in Canada, requiring all health care organizations to recommend reducing in-person visits by $50 \%$. Most provinces in Canada encountered two waves of the pandemic by

\section{Kaitlyn Howden and Adam P. Yan have contributed equally}

Sapna Oberoi

soberoi@cancercare.mb.ca

Extended author information available on the last page of the article
February 2021 and implemented tight restrictions on social gatherings and travel, stay-at-home orders, and closure of non-essential businesses [5]. Before the availability of COVID-19 vaccines, social distancing was one of the only effective measures for mitigating the spread of COVID-19 [6]. Physical distancing has been particularly vital for individuals with cancer due to the heightened risk of COVID19 infection-related morbidity and mortality [7]. However, physical distancing measures may put them at increased risk of experiencing social isolation and loneliness [8-10].

Loneliness is defined as "a perception of being alone and isolated", with this perception mattering more than whether the individual is physically separated from others [11]. Loneliness is not uncommon among patients with cancer and cancer survivors. Cancer and its treatment can often increase loneliness by impacting one's ability to participate in social 
activities, leading to limited shared experiences with peers and inadequate social support [12-14]. The COVID-19 pandemic may have further exacerbated social isolation and loneliness by preventing patients with cancer from being with their loved ones both in and out of the hospital [14]. Loneliness can lead to impaired physical, emotional, and cognitive health in the long term. Therefore, interventions targeting loneliness among patients with cancer are essential $[9,15,16]$.

Adolescents and young adults (AYAs) with cancer are a subgroup of patients diagnosed with cancer between ages 15 and 39. Their unique developmental, social, and emotional needs may put them at greater risk of encountering loneliness than older adults with cancer [15]. For AYAs to become well-functioning individuals, they need to establish their own identity and independence [17]. Social interactions heavily influence this process [17]. While a cancer diagnosis and treatment already disrupt their social maturation, the isolating nature of a pandemic has further put their developmental trajectory at risk [18]. Loneliness emerged as a common theme in a survey of 177 AYAs with cancer that was conducted during the early phases of the pandemic [18]. A recent study of adults with cancer from the USA also identified that $53 \%$ were feeling lonely during this pandemic. However, the older age of the study population (median age $=62.7$ years) and the inclusion of mostly females with breast cancer limited the generalizability of that study to AYAs with cancer, who have different social and developmental needs and cancer types and treatments compared to older adults [18, 19]. Quantifying loneliness experienced by AYAs with cancer and determining which AYAs with cancer are at greater risk of experiencing loneliness during this pandemic can provide vital information to address this unmet need.

Therefore, we aimed to determine the prevalence of loneliness among AYAs with cancer during the COVID19 pandemic, and identify sociodemographic, cancer-, and health-related correlates of loneliness. We hypothesized that the prevalence of loneliness will be higher among AYAs with cancer during this pandemic, and it will be associated with various sociodemographic, cancer-, and health-related variables.

\section{Methods}

\section{Survey design and sample}

The data for this study were obtained through a national cross-sectional survey that sought to analyze the impact of COVID-19 on the health and cancer care of AYAs with cancer living in Canada (ICOVIDAYA). AYAs $\geq 18$ years old, diagnosed with any type of cancer between the age of 15 and 39 years, on or off cancer treatment, living in Canada at the time of survey completion, were eligible to participate in this study. For this analysis, we only included AYAs who were between 18 and 39 years of age at the time of survey completion. Eligible participants completed a 49-item selfadministered anonymous online questionnaire which was offered in both English and French languages. The questionnaire was developed by a study team using an established survey development methodology [20]. The survey took approximately $10-15 \mathrm{~min}$ to complete; the survey items relevant to this analysis are described in the supplemental appendix. Study approval was obtained from the Research Ethics Board at the University of Manitoba (HS: 24501).

\section{Survey administration and setting}

The creation and administration of our survey was accomplished using REDCap, which is a secure web-based application [21]. We recruited participants from all Canadian Provinces and Territories by sharing the online survey link through social media sites of various Canadian AYA support groups such as the Young Adult Cancer Canada (YACC), Pink Pearl Foundation, AYA program at Princess Margaret Cancer Centre, CancerCare Manitoba, Team Shan, VOBOC Foundation (Montreal), and AYA Canada. The survey posters were also displayed at the paediatric and adult oncology clinics at the CancerCare Manitoba to recruit participants. The survey was open between January and February 2021 and required the completion of an online consent form prior to participation (supplemental appendix 1). At the end of survey completion, participants were offered an e-gift card valued at $10 \mathrm{CAD} \$$, with contact details provided for this purpose not being linkable to the survey responses.

\section{Survey measures}

\section{Loneliness}

To assess participants' experience of social isolation over the last 4 weeks, the 3 -item UCLA Loneliness Scale was administered. The 3-item UCLA Loneliness Scale has displayed satisfactory reliability (alpha coefficient of reliability $=0.72$ ) and both concurrent and discriminant validity (correlation with the revised UCLA [R-UCLA] full loneliness scale $=0.82$ ) for measuring loneliness in large population-based surveys [22]. The scale consists of three questions assessing how often participants feel that they lack companionship, feel left out, and feel isolated from others. Response options to each question consist of "hardly ever", "some of the time", or "often", which are scored as 1,2, and 3, respectively. The total score from the three questions is combined to yield a final loneliness score with total scores $\leq 5$ classified as not lonely and scores $\geq 6$ classified as lonely [23]. 


\section{Demographics and clinical variables}

The demographic, cancer-, and health-related information included age, gender, ethnicity, geographic location, relationship status, the impact of COVID-19 on employment, education status, personal income in the year 2020, cancer type, time since cancer diagnosis, current status of cancer treatment, presence of pre-pandemic mental health and chronic physical health conditions, and changes to substance use during the pandemic.

\section{Statistical analysis}

Demographics and loneliness were summarized using descriptive statistics. The prevalence of loneliness was calculated with 95\% confidence intervals (CI) using the binomial distribution. The association of pre-selected variables such as age, gender, ethnicity, geographic location, relationship status, the impact of COVID-19 on employment, personal income in the year 2020, education status, cancer type, time since cancer diagnosis, the current status of cancer treatment, presence of self-reported pre-pandemic mental health condition, pre-existing self-reported chronic physical health condition, relationship status, and changes to substance use during the pandemic compared to pre-pandemic years with the presence of loneliness was tested using chi-square testing and simple logistic regression. These variables were chosen based on the pre-published conceptual frameworks outlining the factors influencing loneliness among patients with or without cancer [11, 14]. The associations were reported using odds ratios (ORs) and 95\% confidence intervals (CI). The factors showing statistically significant association with loneliness on univariable analysis $(p<0.05)$ were subjected to multivariable logistic regression in a step wise manner to determine their independent association with the presence of loneliness (yes vs. no). Correlation between the variables entered in the multiple logistic regression model was examined using correlation matrix and variable inflation factor; no significant correlation existed between these variables (correlation coefficient $<0.35$ and variable inflation factor $<1.2$ for all variables). Missing data were between 0.1 and $2 \%$ for included variables. The participants with missing information on the variables included in the multivariable regression model were not included in the analysis. The $p$-values less than 0.05 were considered statistically significant, and all tests were two-sided. SPSS version 28.0 was used for the analysis [24].

\section{Sample size}

Approximately 8000 AYAs with a cancer diagnosis are engaged in the AYA support networks across which this survey was distributed. Based on the estimated loneliness prevalence of at least 50\% among AYAs with cancer during this pandemic, we determined that the number of individuals required to determine the prevalence of loneliness with a $95 \%$ confidence interval with 5\%, 4\%, and 3\% margin of error will be 368,560 , and 943 , respectively [25].

\section{Results}

\section{Demographic data}

Of the 1063 individuals who completed the survey, 805 were eligible for analysis. We excluded 258 individuals for being over 39 years of age or for not reporting their age. Table 1 displays demographic and clinical data for the study cohort. Individuals between 26 and 39 years of age comprised $78.5 \%(n=632)$ of the cohort. The cohort was relatively balanced between men and women at 55.5\% $(n=445)$ and $44.5 \%(n=357)$, respectively, and had diverse representation from all Canadian Provinces and Territories. While our survey was offered in English and French, the vast majority $(n=799)$ chose to complete the English version. Nearly one-fourth $(N=196,24.5 \%)$ of participants resided in rural/ remote areas. Two-thirds of the participants were in some form of relationship at the time of survey completion, including common law or married $(n=460,57.4 \%)$ or in another type of committed relationship $(n=24,3.0 \%)$. Almost onethird of participants were single $(n=283,35.3 \%)$, with the remainder either being separated or divorced $(n=33,4.1 \%)$ or widowed $(n=4,0.5 \%)$. Thirty-three percent $(n=265)$ of participants were receiving active cancer treatment, while 67.0\% $(n=538)$ had completed their cancer treatment.

\section{Prevalence of loneliness}

Responses to loneliness questions are illustrated in Fig. 1. Three hundred forty-nine (43.4\%) of participants answered "often" for at least one of the three loneliness questions, and $24(3.0 \%)$ answered "often" to all three questions. Using the total loneliness score, the prevalence of loneliness among the study participants was $52.2 \%(N=419,95 \%$ CI 48.7 to $55.6 \%)$.

\section{Factors associated with loneliness}

On univariable analysis, current age of 18-25 years, urban living environment, not in a relationship (single), income in the year $2020 \geq 60,000 \$$, active cancer treatment status, the presence of a self-reported pre-pandemic mental health condition, and presence of a chronic physical health condition were associated with loneliness among AYAs with cancer (all $p<0.05$ ) (Table 2). 
Table 1 Demographic and clinical characteristics of the study population $(n=805)$

\begin{tabular}{|c|c|c|}
\hline Study variable & Number & Percentage \\
\hline \multicolumn{3}{|l|}{ Age } \\
\hline $18-25$ & 173 & $21.5 \%$ \\
\hline $26-39$ & 632 & $78.5 \%$ \\
\hline \multicolumn{3}{|l|}{ Gender $^{\mathrm{a}}$} \\
\hline Male & 445 & $55.5 \%$ \\
\hline Female & 357 & $44.5 \%$ \\
\hline \multicolumn{3}{|l|}{ Ethnicity } \\
\hline Non-white & 35 & $4.4 \%$ \\
\hline White & 770 & $95.6 \%$ \\
\hline \multicolumn{3}{|l|}{ Province/territory } \\
\hline Prairies $^{\mathrm{b}}$ & 233 & $28.9 \%$ \\
\hline Central Canada ${ }^{c}$ & 222 & $27.6 \%$ \\
\hline Atlantic $^{\mathrm{d}}$ & 169 & $21.0 \%$ \\
\hline Territories $^{\mathrm{e}}$ & 93 & $11.6 \%$ \\
\hline British Columbia & 88 & $10.9 \%$ \\
\hline \multicolumn{3}{|l|}{ Living environment ${ }^{\mathrm{f}}$} \\
\hline Urban & 605 & $75.5 \%$ \\
\hline Rural/remote & 196 & $22.5 \%$ \\
\hline \multicolumn{3}{|c|}{ Impact of COVID-19 on employment ${ }^{\mathrm{g}}$} \\
\hline No impact & 174 & $24.0 \%$ \\
\hline Quit or laid off & 550 & $76.0 \%$ \\
\hline \multicolumn{3}{|l|}{ Income in the year $2020^{\mathrm{h}}$} \\
\hline Less than $\$ 40,000$ & 172 & $22.7 \%$ \\
\hline$\$ 40,000$ to less than $\$ 60,000$ & 195 & $25.8 \%$ \\
\hline$\$ 60,000$ or more & 389 & $51.5 \%$ \\
\hline \multicolumn{3}{|l|}{ Education status } \\
\hline In school & 99 & $12.3 \%$ \\
\hline Not in school & 706 & $87.7 \%$ \\
\hline \multicolumn{3}{|l|}{ Cancer type } \\
\hline Hematologic & 155 & $19.3 \%$ \\
\hline Non-hematologic & 650 & $80.7 \%$ \\
\hline \multicolumn{3}{|l|}{ Time since initial cancer diagnosis } \\
\hline$>5$ years & 100 & $12.4 \%$ \\
\hline $2-5$ years & 459 & $57.0 \%$ \\
\hline$<2$ years & 246 & $30.6 \%$ \\
\hline \multicolumn{3}{|l|}{ Cancer treatment status ${ }^{\mathrm{i}}$} \\
\hline Ongoing & 265 & $33.0 \%$ \\
\hline Completed & 538 & $77.0 \%$ \\
\hline \multicolumn{3}{|c|}{ Pre-pandemic mental health condition ${ }^{j}$} \\
\hline No & 682 & $85.2 \%$ \\
\hline Yes & 118 & $14.8 \%$ \\
\hline \multicolumn{3}{|c|}{ Presence of chronic physical health condition } \\
\hline No & 613 & $76.1 \%$ \\
\hline Yes & 192 & $23.9 \%$ \\
\hline \multicolumn{3}{|l|}{ Relationships status $^{\mathrm{a}}$} \\
\hline Single & 318 & $39.8 \%$ \\
\hline In a relationship & 484 & $60.2 \%$ \\
\hline
\end{tabular}

Table 1 (continued)

\begin{tabular}{lll}
\hline Study variable & Number & Percentage \\
\hline Substance use during the pandemic & \\
$\quad$ Better & & \\
$\quad$ Same & 296 & $50.0 \%$ \\
$\quad$ Worse & 227 & $38.4 \%$ \\
Loneliness & 68 & 11.5 \\
$\quad$ No & & \\
$\quad$ Yes & 382 & $47.8 \%$ \\
\hline
\end{tabular}

${ }^{\mathrm{a}} n=802,{ }^{\mathrm{b}}$ Alberta, Manitoba, Saskatchewan, ${ }^{\mathrm{c}}$ Ontario, Quebec, ${ }^{\mathrm{d}} \mathrm{New}$ Brunswick, Nova Scotia, Newfoundland and Labrador, ${ }^{\mathrm{e}}$ Yukon, Northwest Territories, Nunavut, ${ }^{\mathrm{f}} n=801,{ }^{\mathrm{g}} n=724,{ }^{\mathrm{h}} n=756,{ }^{\mathrm{i}} n=803$, ${ }^{\mathrm{j}} n=800,{ }^{\mathrm{k}} n=501$

In multivariable analysis, participants were more likely to have feelings of loneliness if they were 18-25 years old (adjusted OR (AOR) 1.60, CI 1.03-2.47, $p=0.035$ ), currently undergoing cancer therapy (AOR $1.46,95 \%$ CI 1.03-2.07, $p=0.035)$, self-disclosed the presence of a pre-pandemic mental health condition (AOR 2.09, 95\% $\mathrm{CI}=1.22-3.58, p=0.007)$, or were not in a relationship (AOR 2.22, 95\% CI 1.57-3.14, $p<0.001$ ) (Table 2). Participants who lived in rural or remote locations were less likely to experience loneliness (AOR 0.59, 95\% CI 0.40-0.87, $p=0.008$ ) than those living in the urban setting.

\section{Discussion}

This is the first study to evaluate the self-reported loneliness and correlates of loneliness in 805 AYAs with cancer who were either undergoing active cancer therapy or had completed cancer therapy during the COVID-19 pandemic. We identified that nearly half of the participants $(52.2 \%)$ met the criteria for loneliness using the UCLA Loneliness Scale. Although this proportion is comparable to a prior study conducted on older adults with cancer during this pandemic, it is higher than the loneliness prevalence of $21-41 \%$ reported by pre-pandemic studies conducted among adults with cancer $[18,19,26,27]$. This proportion is also substantially higher than the $29 \%$ prevalence of loneliness reported among young adults (18-39 years) during the COVID-19 pandemic by a survey conducted by the Centre for Addiction and Mental Health (CAMH) in November 2020 in Canada [28]. Due to underlying immune suppression heightening the risk for COVID-19-associated complications, patients with cancer have frequently been isolating themselves from others regardless of existent public health restrictions, including from family and friends, than those without cancer $[7,18]$. 


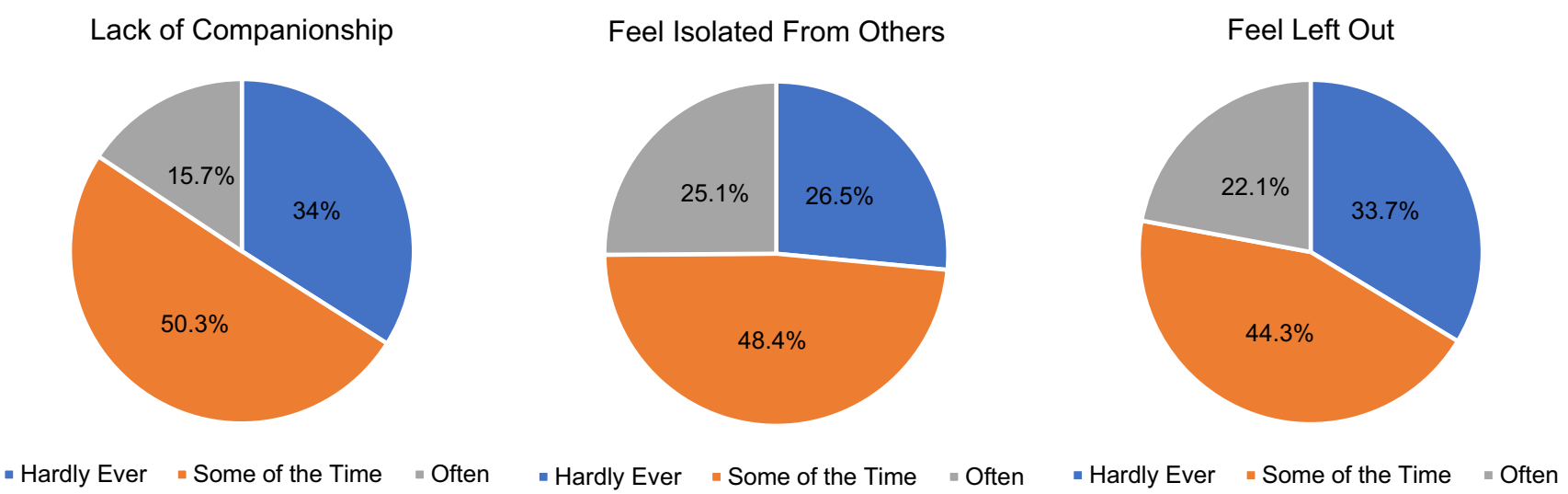

Fig. 1 Responses to individual questions of the 3-item UCLA loneliness scale reported as the percentage of participants

Therefore, they may experience a prolonged and intense period of loneliness than others, often compounded by a pre-existent higher degree of loneliness caused by their cancer diagnosis.

The presence of high degrees of loneliness and social isolation has been a longstanding issue in AYAs with cancer, but the rising rates during the pandemic as highlighted by our study demonstrate the particular vulnerability of this population to the situations that limit their ability to maintain social connectivity [18, 19, 26, 27]. As most AYAs with cancer live 50-60 years past their cancer diagnosis with current treatment regimens, and that self-reported loneliness has been associated with increased morbidity and mortality, it is concerning that so many AYAs with cancer identified as lonely in our study $[9,15]$. Understanding the factors associated with increased loneliness in AYAs with cancer during the pandemic may help develop strategies to improve the quality of life for this population, both during the remainder of the pandemic and into the post-pandemic era.

Participants in the 18-25 age group were more likely to experience loneliness than those aged 26-39. The AYA category encompasses a wide age range. While individual trajectories can vary, most individuals have established their identity, career, and relationships when they reach the older end of the AYA age bracket $[29,30]$. AYAs between the ages of 18-25 are more likely to be in post-secondary education or at the early stages of career development, financially dependent on their families, and often are not married or in a long-term committed relationship compared to those aged 26-39 [29, 30]. The challenges in accomplishing the age-based norms due to the combination of cancer and the pandemic might have contributed towards the increased feeling of loneliness among these younger AYAs.

Individuals undergoing active cancer treatment were more likely to identify as lonely than those who had completed therapy in our study. Individuals on active therapy are more likely to require frequent hospital visits and potential admissions to the hospital. To reduce the risk of in-hospital COVID-19 transmission, many hospitals have either limited or entirely prohibited patients from having any visitors in the hospital. The importance of family members or other supportive companions in a cancer patients' journey is well-established [31-33]. Family members or designated support persons provide critical emotional support and companionship for patients; their involvement in making medical decisions has been associated with reduced anxiety and depression among patients [31-33]. While limited, the existing evidence does not support that the designated visitors increase the hospital-related transmission of COVID$19[34,35]$. Given the profound impact of limiting visitations on patients with cancer, hospitals should implement the least restrictive visitation policies that maintain a safe patient care environment and creatively engage patients and their families to combat their feeling of loneliness. This may include limiting the individuals allowed to visit the patients throughout hospitalization, screening for COVID-19 infection before visiting, checking COVID-19 vaccination status, and using appropriate personalized protective equipment [35].

Higher rates of mental health issues have been a significant concern during the COVID-19 pandemic for individuals with and without cancer. Loneliness during the pandemic has been associated with more significant depressive symptoms and suicidal ideation in both groups $[9,36]$. Among 518 people with cancer in the UK, those who reported loneliness during the pandemic had a 4.5 -fold increased risk of depression [9]. In our study, individuals with a pre-existing mental health condition reported greater loneliness than those without a pre-existing mental health condition. It is plausible that those with a previous mental health conditions are more vulnerable to the social isolation of the pandemic due to a lack of existing coping mechanisms or the inability to access their usual coping mechanisms such as spending 
Table 2 Factors associated feelings of loneliness among AYAs with cancer

\begin{tabular}{|c|c|c|c|c|c|c|}
\hline \multirow[t]{2}{*}{ Study variable } & \multicolumn{2}{|c|}{ Univariable analysis } & \multicolumn{4}{|c|}{ Multivariable analysis $(n=678)$} \\
\hline & Odds ratio & $95 \% \mathrm{CI}$ & $P$-value & $\begin{array}{l}\text { Adjusted } \\
\text { odds } \\
\text { ratio }\end{array}$ & $95 \% \mathrm{CI}$ & $P$-value \\
\hline \multicolumn{7}{|l|}{ Age } \\
\hline $\begin{array}{l}18-25 \\
26-39 \text { (ref) }\end{array}$ & 1.41 & $1.00-1.98$ & 0.049 & 1.60 & $1.03-2.47$ & 0.035 \\
\hline \multicolumn{7}{|l|}{ Gender } \\
\hline $\begin{array}{l}\text { Female } \\
\text { Male (ref) }\end{array}$ & \multicolumn{5}{|c|}{ Male (ref) } & \\
\hline \multicolumn{7}{|l|}{ Ethnicity } \\
\hline \multicolumn{7}{|l|}{ White (ref) } \\
\hline \multicolumn{7}{|l|}{ Living environment } \\
\hline $\begin{array}{l}\text { Rural/remote } \\
\text { Urban (ref) }\end{array}$ & 0.67 & $0.48-0.92$ & 0.014 & 0.59 & $0.40-0.87$ & 0.008 \\
\hline \multicolumn{7}{|c|}{ Impact of COVID-19 on employment } \\
\hline $\begin{array}{l}\text { Quit or laid off } \\
\text { No impact (ref) }\end{array}$ & \multicolumn{5}{|c|}{ No impact (ref) } & \\
\hline \multicolumn{7}{|l|}{ Income in the year 2020} \\
\hline$\$ 60,000$ or more & 1.14 & $0.75-1.74$ & 0.046 & 1.17 & $0.72-1.90$ & 0.53 \\
\hline \multicolumn{7}{|l|}{ Less than $\$ 40,000$ (ref) } \\
\hline \multicolumn{7}{|l|}{ Education status } \\
\hline \multicolumn{6}{|l|}{ Not in school (ref) } & \\
\hline \multicolumn{7}{|l|}{ Cancer type } \\
\hline \multicolumn{7}{|l|}{ Non-hematologic (ref) } \\
\hline \multicolumn{7}{|l|}{ Time since initial cancer diagnosis } \\
\hline$>5$ years & 0.91 & $0.59-1.41$ & 0.67 & & & \\
\hline $2-5$ years & 1.24 & $0.78-1.98$ & 0.36 & & & \\
\hline \multicolumn{7}{|l|}{$<2$ years (ref) } \\
\hline \multicolumn{7}{|l|}{ Cancer treatment status } \\
\hline \multicolumn{6}{|l|}{ Completed (ref) } & 0.035 \\
\hline \multicolumn{7}{|c|}{ Pre-pandemic mental health condition } \\
\hline \multicolumn{6}{|l|}{ No (ref) } & 0.007 \\
\hline \multicolumn{7}{|c|}{ Presence of chronic physical health condition } \\
\hline \multicolumn{7}{|l|}{ No (ref) } \\
\hline \multicolumn{7}{|l|}{ Relationships status } \\
\hline $\begin{array}{l}\text { Single } \\
\text { In relationship (ref) }\end{array}$ & 1.88 & $1.41-2.51$ & $<0.001$ & 2.22 & $1.57-3.14$ & $<0.001$ \\
\hline Substance use during the panden & & & & & & \\
\hline Better & 1.07 & $0.76-1.51$ & 0.71 & & & \\
\hline Worse & 0.69 & $0.40-1.20$ & 0.19 & & & \\
\hline Don’t use & 1.02 & $0.70-1.49$ & 0.92 & & & \\
\hline Same (ref) & & & & & & \\
\hline
\end{tabular}


time with their friends and family, going outdoors, exercising, and accessing mental health supports.

Both before and during the pandemic, being unmarried or unpartnered was identified as a significant risk factor for loneliness among individuals with cancer [13, 19]. This association was held in our study, with participants not currently in a relationship being two times more likely to experience loneliness than those in a relationship. Individuals in a relationship may have had more companionship during public health mandated stay-at-home orders, reducing their reported loneliness compared to those who are single. The pandemic restrictions have made it challenging to meet other individuals and form new relationships, particularly those who struggle to make connections through virtual platforms or do not have access to the technology required to utilize social media [37]. It may take time once the pandemic is over for AYAs, especially those with cancer, to find opportunities to rebuild their social network, which puts them at risk of experiencing loneliness over a prolonged period.

Individuals living in rural and remote Canadian locations were $40 \%$ less likely to report loneliness than those living in urban locations. Regional differences in the burden of COVID-19 cases, duration of lockdowns, and degree of restrictions imposed by public health orders may have resulted in different social isolation restrictions in different locations in Canada. For instance, Ontario's largest city, Toronto, had a higher incidence of COVID-19 and more extended lockdown periods than Northern Ontario, resulting in tighter restrictions which may have increased loneliness in this urban location [38]. Also, the lockdowns also limited access to several social activities that are more readily available to those living in the urban setting than those in rural settings $[39,40]$. The sense of community and belonging can be more strongly developed in rural areas due to smaller population sizes than larger urban areas; this increased connectivity may protect against loneliness through increased access to social support [41]. Whether geographic location affects the feelings of loneliness among AYAs with cancer in the post-pandemic era should be explored in future studies. We also found that nearly three-quarters of participants were laid off or quit their job during this pandemic. Although not found to be significantly associated with loneliness, unemployment might have increased feelings of loneliness due to lack of opportunities to socialize with others in the workplace [42].

The results of our study should be considered in light of its limitations. Caution must be taken when generalizing these results to the larger population of AYAs with cancer, as there may be key differences between the two groups. For instance, very few participants in our study were identified as non-white or belonging to a gender minority group $(n=35)$. Given that COVID-19 has disproportionately impacted AYAs of colour or gender diverse AYAs, their experience of loneliness during the pandemic may differ from that of our cohort [43]. Therefore, future studies should evaluate loneliness among this subgroup of AYAs in greater detail. Our study was also conducted online, which may have impaired individuals with limited access to technology from participating, including those from rural or remote areas. The use of self-reported outcomes may have also introduced bias to the study. Other confounding factors such as highest level of education, access to digital technology or social media, time spent on social media, and the number of people in the household, quality of social interactions, not measured in this study, could have also altered the association of examined factors with the loneliness among AYAs with cancer. Finally, the dynamic state of pandemic and public health care restrictions may limit the generalizability of the results for other time points.

Many parts of the world remain in lockdown, and the potential for future lockdowns prevails with the emergence of new, more contagious, and lethal COVID-19 variants [44]. With further lockdowns, the incidence and severity of loneliness may rise. It is therefore pivotal to develop strategies to combat loneliness amongst individuals with cancer. Studies conducted pre-pandemic reported psychological therapies such as mindfulness-based interventions, cognitive behaviour therapy (CBT), visual art discussions, and social facilitation interventions enhancing social support to be effective in reducing loneliness or social isolation among diverse groups of adults [45-48]. CBT was also found to be an effective tool for mitigating loneliness among elderly individuals during COVID-19 pandemic [49]. As the data on the efficacy of these interventions among AYAs with cancer is lacking, future studies should evaluate their impact on the loneliness of this population. These interventions should be specifically targeted for the AYAs identified at increased risk of loneliness. Also, due to the dynamic nature of this pandemic and ever-changing public health restrictions, longitudinal studies are necessary to assess how loneliness varies among this population and how they cope with loneliness.

In conclusion, we demonstrated that one in two AYAs with cancer suffer from loneliness during the COVID-19 pandemic. We identified active cancer treatment status, being single, presence of pre-pandemic mental health issues, and urban living environment as the independent factors associated with loneliness. Future strategies to prevent and mitigate loneliness among AYAs with cancer are urgently needed as we continue to battle against this pandemic, prepare for future pandemics, and to improve the overall health and well-being of AYAs with cancer.

Supplementary Information The online version contains supplementary material available at https://doi.org/10.1007/s00520-021-06628-5. 
Acknowledgements We want to thank our study participants and patient partners for their contribution to this study.

Author contribution Conception and design: All authors.

Collection and assembly of data: Adam Yan, Kaitlyn Howden, Camille Glidden, Sapna Oberoi.

Data analysis and interpretation: Adam Yan, Kaitlyn Howden, Camille Glidden, Sapna Oberoi.

Manuscript writing: Adam Yan, Kaitlyn Howden and Sapna Oberoi. Final approval of manuscript: All authors.

Accountable for all aspects of the work: All authors.

Funding This work was funded by CancerCare Manitoba Foundation (Grant number: 764135447).

Data availability The datasets generated during and/or analyzed during the current study are available from the corresponding author on reasonable request.

Code availability Codes used to analyze the data are available from the corresponding author on reasonable request.

\section{Declarations}

Ethics approval This study was approved by the University of Manitoba Health Research Ethics Board (HS: 24501). The study was performed in accordance with the ethical standards as laid down in the 1964 Declaration of Helsinki and its later amendments or comparable ethical standards.

Consent to participate The participants provided informed consent online before completing the survey questionnaire.

Consent for publication Not applicable.

Competing interests The authors declare no competing interests.

\section{References}

1. WHO Coronavirus (COVID-19) Dashboard. World Health Organization. Published July 2, 2021. Accessed July 2, 2021. https:// covid19.who.int/

2. Honey-Roses J, Anguelovski I, Chireh V, et al (2020) The impact of COVID-19 on public space: an early review of the emerging questions - design, perceptions and inequities. Cities Health. 1-17. https://doi.org/10.1080/23748834.2020.1780074

3. Momtazmanesh S, Samieefar N, Uddin LQ et al (2021) Socialization during the COVID-19 pandemic: the role of social and scientific networks during social distancing. Adv Exp Med Biol 1318:911-921. https://doi.org/10.1007/978-3-030-63761-3_51

4. Ward P (2020) A sociology of the Covid-19 pandemic: a commentary and research agenda for sociologists. J Sociol 56(4):726-735. https://doi.org/10.1177/1440783320939682

5. COVID-19 Daily Epidemiology Update. Government of Canada. Published August 27, 2021. Accessed August 28, 2021. https:// health-infobase.canada.ca/covid-19/epidemiological-summarycovid-19-cases.html\#a4

6. Koh W, Naing L, Wong $\mathbf{J}$ (2020) Estimating the impact of physical distancing measures in containing COVID-19: an empirical analysis. Int J Infect Dis 100:42-49. https://doi.org/10.1016/j.ijid. 2020.08.026
7. Fung M, Babik J (2021) COVID-19 in immunocompromised hosts: what we know so far. Clin Infect Dis 72(2):340-350. https:// doi.org/10.1093/cid/ciaa863

8. Schellekens M, van der Lee M (2020) Loneliness and belonging: exploring experiences with the COVID-19 pandemic in psychooncology. Psycho-oncol 29:1399-1401. https://doi.org/10.1002/ pon.5459

9. Gallagher S, Bennett K, Roper L (2020) Loneliness and depression in patients with cancer during COVID-19. J Psychosoc Oncol 39(3):445-451. https://doi.org/10.1080/07347332.2020.1853653

10. Younger E, Smrke A, Lidington E, Farag S, Ingley K, Chopra N, Maleddu A, Augustin Y, Merry E, Wilson R, Benson C, Miah A, Zaidi S, McTiernan A, Strauss SJ, Dileo P, Gennatas S, Husson O, Jones RL (2020) Health-related quality of life and experiences of sarcoma patients during the COVID-19 pandemic. Cancers (Basel) 12(8):2288. https://doi.org/10.3390/ cancers 12082288

11. Tiwari S (2013) Loneliness: a disease? Indian J Psychiatry 55(4):320-322. https://doi.org/10.4103/0019-5545.120536

12. Rosedale M (2009) Survivor loneliness of women following breast cancer. Oncol Nurs Forum 36(2):175-183. https://doi. org/10.1188/09.ONF.175-183

13. Deckx L, van den Akker M, Buntinx F (2014) Risk factors for loneliness in patients with cancer: a systematic literature review and meta-analysis. Eur J Oncol Nurs 15(5):466-477

14. Adams R, Mosher C, Abonour R et al (2016) Cognitive and situational precipitants of loneliness among patients with cancer: a qualitative analysis. Oncol Nurs Forum 43(2):156-163. https:// doi.org/10.1188/16.ONF.156-163

15. Islam JY, Vidot DC, Camacho-Rivera M (2021) Evaluating mental health-related symptoms among cancer survivors during the covid-19 pandemic: an analysis of the covid impact survey. JCO Oncology Practice 17:9, e1258-e1269

16. Masi CM, Chen HY, Hawkley LC, Cacioppo JT (2011) A metaanalysis of interventions to reduce loneliness. Pers Soc Psychol Rev 15(3):219-266. https://doi.org/10.1177/1088868310377394

17. Sanders R (2013) Adolescent psychosocial, social, and cognitive development. PIR 34(8):354-359. https://doi.org/10.1542/pir. 34-8-354

18. Košir U, Loades M, Wild J et al (2020) The impact of COVID19 on the cancer care of adolescents and young adults and their well-being: results from an online survey conducted in the early stages of the pandemic. Cancer 126(19):4414-4422. https://doi. org/10.1002/cncr.33098

19. Miaskowski C, Paul SM, Snowberg K, Abbott M, Borno HT, Chang SM, Chen LM, Cohen B, Cooper BA, Hammer MJ, Kenfield SA, Kober KM, Laffan A, Levine JD, Pozzar R, Rhoads K, Tsai KK, Van Blarigan EL, Van Loon K (2021) Loneliness and symptom burden in oncology patients during the COVID-19 pandemic. Cancer 127(17):3246-3253. https://doi.org/10.1002/cncr.33603

20. Millar M, Dillman D (2011) Improving response to web and mixed-mode surveys. Public Opin Q 75(2):249-269. https://doi. org/10.1093/poq/nfr003

21. Harris P, Taylor R, Thielke R, Payne J, Gonzaolez N, Conde J (2009) Research electronic data capture (REDCap) - A metadata-driven methodology and workflow process for providing translational research informatics support. J Biomed Inform 42(2):377-381. https://doi.org/10.1016/j.jbi.2008.08.010

22. Hughes M, Waite L, Hawkley L, Cacioppo J (2004) A short scale for measuring loneliness in large surveys. Res Aging 26(6):655-672. https://doi.org/10.1177/0164027504268574

23. Steptoe A, Shenkar A, Demakakos P, Wardle J (2013) Social isolation, loneliness, and all-cause mortality in older men and women. PNAS 110(15):5797-5801. https://doi.org/10.1073/ pnas. 1219686110 
24. IBM Corp. Released (2020) IBM SPSS Statistics for Windows, Version 28.0. Armonk, NY: IBM Corp

25. Sergeant, ESG, 2018. Epitools Epidemiological Calculators. Ausvet. Available at: http://epitools.ausvet.com.au. Accessed 10 Nov 2020

26. Deckx L, van den Akker M, van Driel M et al (2015) Loneliness in patients with cancer: the first year after cancer diagnosis. Psycho-Oncol 24:1521-1528. https://doi.org/10.1002/pon.3818

27. Yildirim Y, Kocabiyik S (2009) The relationship between social support and loneliness in Turkish patients with cancer. J Clin Nurs 19:832-839. https://doi.org/10.1111/j.1365-2702.2009. 03066.X

28. COVID-19 National Survey Dashboard. The Centre for Addiction and Mental Health. Published April 2021. Accessed July 2, 2021. https://www.camh.ca/en/health-info/mental-health-and-covid-19/ covid-19-national-survey

29. Shovestul B, Han J, Germine L, Dodell-Feder D (2020) Risk factors for loneliness: the high relative importance of age versus other factors. PLOS ONE 15(2):e0229087. https://doi.org/10.1371/ journal.pone. 0229087

30. Luhmann M, Hawkley L (2016) Age differences in loneliness from late adolescence to oldest age. Dev Psychol 52(6):943-959. https://doi.org/10.1037/dev0000117

31. Labrecque MS, Blanchard CG, Ruckdeschel JC et al (1991) The impact of family presence on the physician-cancer patient interaction. Soc Sci Med 33(11):1253-1261. https://doi.org/10.1016/ 0277-9536(91)90073-L

32. Royak-Schaler R, Gadalla S, Lemkau J et al (2006) Family perspectives on communication with healthcare providers during end-of-life cancer care. Oncol Nurs Forum 33(4):753-760. https:// doi.org/10.1188/06.ONF.753-760

33. Zhang AY, Siminoff LA (2003) The role of the family in treatment decision making by patients with cancer. Oncol Nurs Forum 30(6):1022-1028. https://doi.org/10.1188/03.ONF.1022-1028

34. Rhee C, Baker M, Vaidya V et al (2020) Incidence of nosocomial COVID-19 in patients hospitalized at a large US academic medical center. JAMA Netw Open 3:e2020498. https://doi.org/ 10.1001/jamanetworkopen.2020.20498

35. Munshi L, Evans G, Razak F (2021) The case for relaxing no-visitor policies in hospitals during the COVID-19 pandemic. CMAJ 193(4):E135-E137. https://doi.org/10.1503/cmaj.202636

36. Killgore W, Cloonan S, Taylor E, Dailey N (2020) Loneliness: a signature of mental health concern in the era of COVID-19. Psychiatry Res 290:113117. https://doi.org/10.1016/j.psychres. 2020.113117

37. Shah S, Nogueras D, van Woerden H, Kiparoglou V (2020) The COVID-19 pandemic: a pandemic of lockdown loneliness and the role of digital technology. J Med Internet Res 22(11):e22287. https://doi.org/10.2196/22287
38. Ontario Agency for Health Protection and Promotion (Public Health Ontario) (2020) Evolution of COVID-19 case growth in Ontario. Toronto, ON: Queen's Printer for Ontario

39. Henning-Smith C, Moscovice I, Kozhimannil K (2019) Differences in social isolation and its relationship to health by rurality. J Rural Health 35:540-549. https://doi.org/10.1111/jrh.12344

40 Bu F, Steptoe A, Fancourt D (2020) Loneliness during a strict lockdown: trajectories and predictors during the COVID-19 pandemic in 38,217 United Kingdom adults. Soc Sci Med 265:113521. https://doi.org/10.1016/j.socscimed.2020.113521

41. Menec V, Newall N, Mackenzie C, Shooshtari S, Nowicki S (2019) examining individual and geographic factors associated with social isolation and loneliness using Canadian Longitudinal Study on Aging (CLSA) Data. PLoS One 14(2):e0211143. https:// doi.org/10.1371/journal.pone.0211143

42. Morrish N, Medina-Lara A (2021) Does unemployment lead to greater levels of loneliness? A systematic review. Soc Sci Med 287:114339. https://doi.org/10.1016/j.socscimed.2021.114339

43. Ruprecht M, Wang X, Johnson A et al (2021) Evidence of social and structural COVID-19 disparities by sexual orientation, gender identity, and race/ethnicity in an urban environment. J Urban Health 98(1):27-40. https://doi.org/10.1007/s11524-020-00497-9

44. Torjesen I (2021) Covid-19: delta variant is now UK's most dominant strain and spreading through schools. BMJ 373:n1445. https://doi.org/10.1136/bmj.n1445

45. Zhang N, Fan F, Huang S, Rodriquez M (2018) Mindfulness training for loneliness among Chinese college students: a pilot randomized controlled trial. Int J Psychol 53(5):373-378. https://doi. org/10.1002/ijop.12394

46. Wikström B-M (2002) Social interaction associated with visual art discussions: a controlled intervention study. Aging Ment Health 6(1):82-87. https://doi.org/10.1080/13607860120101068

47. Czaja S, Boot W, Charness N, Rogers W, Sharit J (2018) Improving social support for older adults through technology: findings from the PRISM randomized controlled trial. Gerontologist 58(3):467-477. https://doi.org/10.1093/geront/gnw249

48. Williams C, Townson A, Kapur M et al (2021) Interventions to reduce social isolation and loneliness during COVID-19 physical distancing measures: a rapid systemic review. PLoS One 16(2):e0247139. https://doi.org/10.1371/journal.pone.0247139

49. Hall B, Short V, Giberson S, Howe-Martin L (2020) A cognitive behavioural therapy group for adolescent and young adult cancer patients: a review of a pilot program. J Adolesc Young Adult Oncol 9(3):422-425. https://doi.org/10.1089/jayao.2019.0104

Publisher's note Springer Nature remains neutral with regard to jurisdictional claims in published maps and institutional affiliations. 


\section{Authors and Affiliations}

\section{Kaitlyn Howden ${ }^{1}$ - Adam P. Yan ${ }^{2}$. Camille Glidden ${ }^{3} \cdot$ Razvan G. Romanescu $^{4} \cdot$ Ian Scott $^{5} \cdot$ Julie M. Deleemans $^{6}$. Karine Chalifour ${ }^{7}$. Geoff Eaton ${ }^{7}$. Abha A. Gupta ${ }^{1,8}$. James M. Bolton ${ }^{3,9}$. Sheila N. Garland ${ }^{10}$. Alyson L. Mahar ${ }^{11,12}$. Sapna Oberoi ${ }^{1,12,13}$}

1 Department of Pediatrics and Child Health, University of Manitoba, Winnipeg, Canada

2 Division of Pediatric Hematology Oncology, The Hospital for Sick Children, University of Toronto, Toronto M5G1X8, Canada

3 Department of Psychiatry, University of Manitoba, Winnipeg, Canada

4 George \& Fay Yee Centre for Healthcare Innovation, University of Manitoba, Winnipeg, Canada

5 Department of Psychosocial Oncology, CancerCare Manitoba, Winnipeg, Canada

6 Division of Oncology, Department of Psychosocial Oncology, University of Calgary Cumming School of Medicine, Calgary, AB, Canada

7 Young Adult Cancer Canada, St. John's, Newfoundland and Labrador, Canada
8 Adolescent and Young Adult Program, Princess Margaret Cancer Care Research Institute, Toronto, ON, Canada

9 Manitoba Centre for Health Policy, University of Manitoba, Winnipeg, Canada

10 Departments of Psychology and Oncology, Memorial University, St. John's, Newfoundland and Labrador, Canada

11 Department of Community Health Sciences, Max Rady College of Medicine, University of Manitoba, Winnipeg, Canada

12 CancerCare Manitoba Research Institute, 675 McDermot Avenue, Winnipeg, Canada

13 Department of Pediatric Hematology-Oncology, CancerCare Manitoba, Winnipeg, Canada 